\title{
Alopezien - Diagnostisches und therapeutisches Management
}

\author{
Alopecia - Diagnostic and Therapeutic Management
}

T. W. Fischer

\section{Lernziele \\ $\nabla$}

Dieser Fortbildungsartikel soll jedem Hautarzt in Weiterbildung einen Kurzüberblick über die Hauptformen der Alopezien geben, aber auch dem bereits langjährig erfahrenen Dermatologen als Auffrischung der aktuellen Erkenntnisse über die verschiedenen Formen der Alopezien dienen sowie seltenere Krankheitsbilder kurz erwähnen, um mit den aktuell verfügbaren evidenzbasierten Therapien abzuschließen. Dabei beschränkt sich der Artikel aufgrund der Umfangslimitation bewusst auf wesentliche Fakten und muss auf Details, die in Übersichtsarbeiten zu den jeweiligen Krankheitsbildern nachzulesen sind, verzichten.

\section{Einleitung}

Haarausfall ist ein immer wieder als „leidiges“ Thema empfundener Krankheitskomplex: leidig einerseits für die betroffenen Patientinnen und Patienten, da diese häufig sehr stark in ihrer Lebensqualität und ihrem Selbstempfinden eingeschränkt sind, und leidig andererseits für die konsultierten Ärzte, da sie häufig einem hohen Erwartungsdruck der Patienten bei gleichzeitig bestehender Begrenztheit effektiver therapeutischer Möglichkeiten ausgesetzt sind. Auch wenn die meisten Therapeutika zur Behandlung des Haarausfalls von den Krankenkassen als „kosmetisch“ bzw. „nicht medizinisch“ eingeordnet werden und daher nicht kostenerstattungfähig sind, liegen bei einer nicht unbeträchlichen Zahl von Patienten erhebliche Probleme vor $[1,2]$. Insofern liegt gemessen an der WHO-Definition für $\mathrm{Ge}-$ sundheit als die vollkommene Intaktheit des körperlichen, geistigen und seelischen Befindens auch bei Haarausfall eine Erkrankung vor. Der Dermatologe als Kompetenzträger für Erkrankungen von Haut und Haar sollte als Behandlungspartner für von Haarausfall betroffene Patienten zuverlässige Kenntnisse der Pathogenese und der klinischen Ausprägung sowie Verlauf der häufigs- ten Formen von Haarausfall haben und die therapeutischen Entscheidungen evidenzbasiert, situationsgerecht und individuell auf den Patienten zugeschnitten treffen, um in einem langfristigen Behandlungsbündnis mit dem Patienten realistische Erfolge zu erzielen. Der aktuelle Fortbildungsartikel soll daher Wissen zur korrekten Einschätzung der verschiedenen Formen des Haarausfalls und deren Behandlung geben.

\section{Einteilung der Alopezien \\ $\checkmark$}

Die Alopezien können prinzipiell in nicht vernarbende (reversible) und vernarbende (irreversible) Alopezien sowie in zirkumskripte und diffuse Formen eingeteilt werden. $\mathrm{Zu}$ den reversiblen Alopezien gehören die Alopecia areata (AA), die symptomatische Alopecia diffusa (AD), bakterielle und mykotische Kopfhautentzündungen, wenn sie oberflächlich sind, und potenziell auch die Alopecia androgenetica (AGA), da keine Vernarbung vorliegt und die Miniaturisierung der betroffenen Haarfollikel bei frühem Therapiebeginn partiell rückgängig gemacht werden kann. Beispiele für irreversible Alopezien umfassen den Lupus erythematodes capillitii, den Lichen ruber follicularis (syn. Lichen planopilaris), die Sklerodermie (v.a. En coup de sabre) und tiefe bakterielle und mykotische Entzündungen i.S. einer Folliculitis decalvans oder einer tiefen Trichophytie.

Grundsätzlich unterscheidet man nicht vernarbende (reversibel) und vernarbende (irreversibel) Alopezien.

\section{Ursachen von Alopezien \\ $\nabla$}

Ursachen für Haarausfall können

- genetische Disposition (AGA)

- autoimmune Reaktionen (AA)

- Medikamenteneinnahme

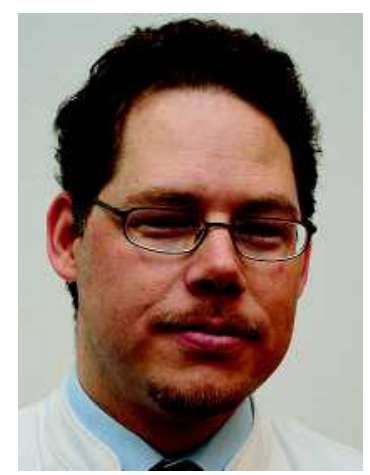

Dr. med. Tobias W. Fischer

Korrespondenzadresse Dr. med. Tobias W. Fischer Klinik für Dermatologie, Allergologie und Venerologie Universitätsklinikum Schleswig-Holstein Universität zu Lübeck Ratzeburger Allee 160 23538 Lübeck

Tobias.Fischer@uk-sh.de

Bibliografie DOI 10.1055/s-2007-995795 Akt Dermatol 2008; 34: 209-225 @ Georg Thieme Verlag KG Stuttgart · New York ISSN 0340-2541 

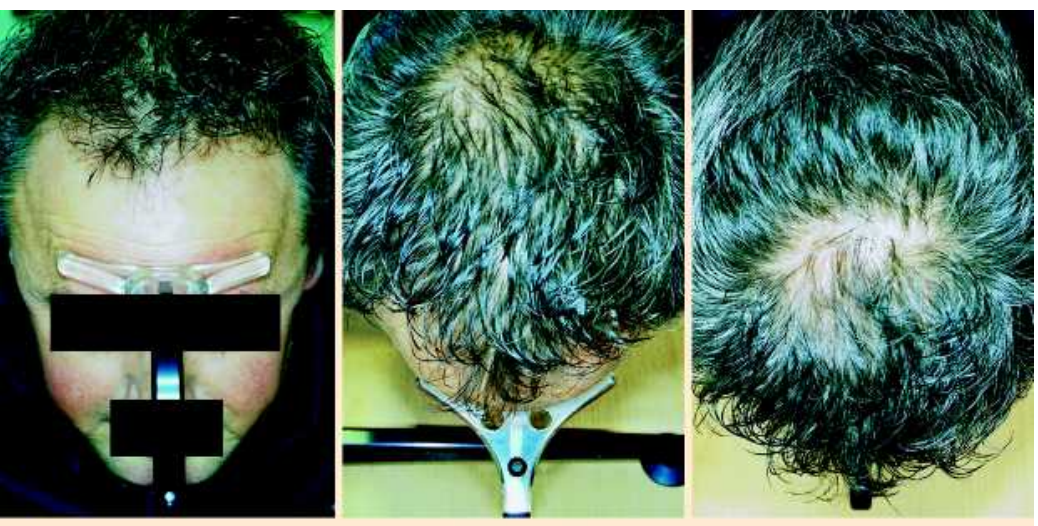

a

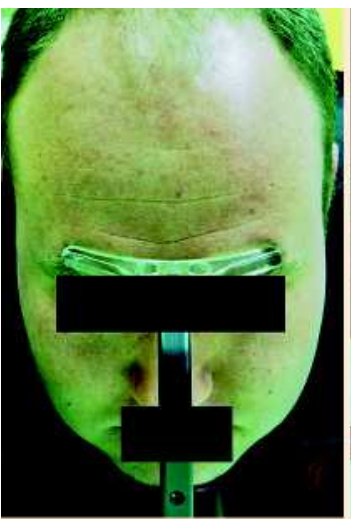

b

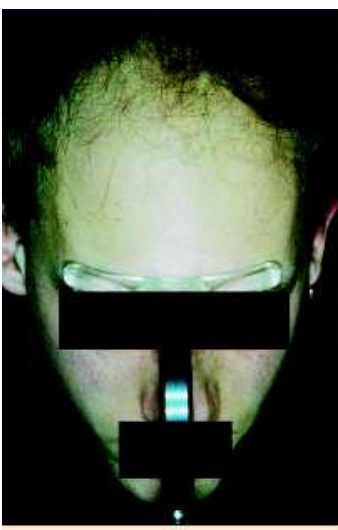

c
Stadium IIV

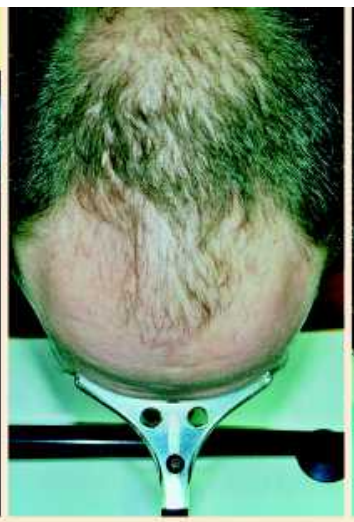

Stadium IV

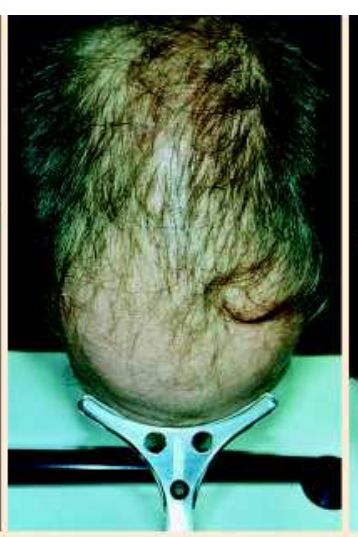

Stadium V
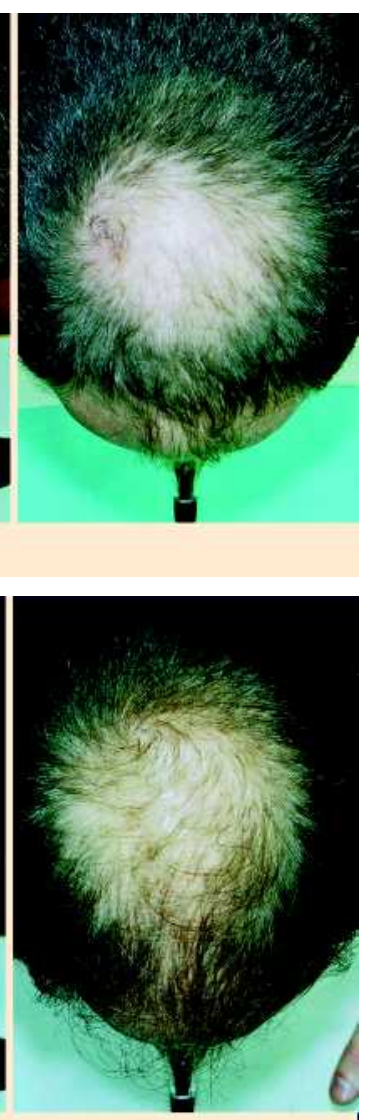

Abb. 1 Klinische Übersichtsfotografien mittels standardisierter Fotografiertechnik (CanfieldTechnik) verschiedener Stadien der androgenetischen Alopezie „male pattern“. Die Stadien werden nach Hamilton in I - VII eingeteilt und betreffen z. B. im Stadium IIv (a) die temporofrontale Region („Geheimratsecken“) sowie beginnend den Vertex-Bereich („"“). Im Stadium IV sind die kahlen Stellen der temporo-frontalen Regionen bereits deutlich nach hinten reichend und das Vertex-Areal ist ebenfalls deutlich gelichtet, sodass lediglich ein relativ dünner Haarsteg in der Transversallinien-Region des Kapillitiums bestehen bleibt (b). Im Stadium V finden sich von frontal und vom Vertex ausgehende konfluierende gelichtete Areale (c).

- Zustand nach Operation/Vollnarkose, Mangelzustände (z. B. Eisenmangel)

> endokrinologische Veränderungen (z.B. Hypo-, Hyperthyreose, Diabetes mellitus)

- systemisch generalisierte oder lokal schwerwiegende Entzündungen (AD z. B. bei systemischem Lupus erythematodes, Lues, schwere Grippe, Pneumonie u.ä.) und
- unausgewogene Ernährung (relativer Eiweißbzw. Vitaminmangel) sein.

\section{Häufige Formen des Haarausfalls}

$\nabla$

Androgenetische Alopezie

Die androgenetische Alopezie (AGA) tritt bei etwa jedem 2. Mann im Laufe des Lebens auf, während bei den Frauen etwa 10-20\% betroffen sind. Pathogenetisch liegt beim Mann eine genetisch determinierte Hypersensitivität von prädisponierten Haarfollikeln in der Schläfen- und Vertexregion auf Androgene (Normalspiegel) vor, die eine Miniaturisierung der Haarfollikel bis zum kompletten Haarverlust bedingt. Beim Mann ist es vor allem die erhöhte Aktivität des Enzyms $5 \alpha$-Reduktase Typ 2, welches intrafollikulär die Umwandlung von im Blut zirkulierendem Testosteron in das Effektor-Hormon Dihydrotestosteron (DHT) katalysiert [3-8]. Darüber hinaus wird ein ursächlicher Zusammenhang mit dem Polymorphismus des Androgenrezeptor-Gens vermutet $[7,9]$.

Verlauf. Der Beginn der AGA kann schon sehr früh im Alter von unter 20 Jahren bis zum 30. Lebensjahr liegen. Typischerweise lichten sich in den frühen Stadien zunächst die Temporalregionen des behaarten Kopfes („Geheimratsecken“). In einigen Fällen kann die AGA in diesem Stadium konstant bleiben, ohne dass es zu einer weiteren Lichtung des Kapillitiums kommt. Bei den meisten Betroffenen tritt aber im späteren Verlauf eine zunehmende Lichtung des Haares im Hinterkopfbereich (Vertex-Region) auf, die dann im weiteren Verlauf mit der immer weiter nach hinten weichenden temporo-frontalen Lichtung konfluiert, bis im maximalen Spätstadium eine Glatze mit einem sich okzipital zwischen beiden Ohren erstreckenden Resthaarkranz resultiert. Die Stadien der AGA beim Mann wurden nach Hamilton und Norwood (1951 und 1975) eingeteilt $[10,11]$ ( $\bullet$ Abb. 1).

Hormonspiegel bei AGA normal; Pathogenese: erhöhte intrafollikuläre $5 \alpha$-Reduktase-Aktivität und Polymorphismus des Androgen-Rezeptor-Gens

Vererbung. Die Vererbung ist multifaktoriell mit inkonstanter Penetranz, wofür es zahlreiche Beispiele aus der klinischen Praxis gibt. Beispielsweise muss weder der Sohn eines Vaters, der an einer AGA leidet, diese entwickeln, noch ist bei vollem Haar des Vaters die Entwicklung einer AGA beim Sohn sicher ausgeschlossen. Die genetischen Faktoren können über mehrere Generationen und auch über die Mutter (z.B. Großvater der Mutter betroffen) vererbt werden. Die beschriebene klinische Ausprägung der AGA beim Mann findet sich als sogenannte Male-Pattern- 


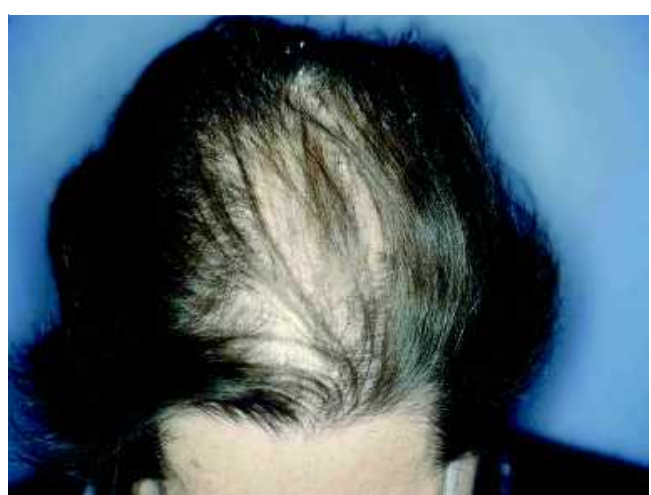

Abb. 2 Klinisches Bild der androgenetischen Alopezie Female Pattern. Die Female-Pattern-Alopezie wird in Stadien nach Ludwig eingeteilt, wobei Stadium I eine diskrete diffuse, jedoch im Oberkopf umschriebene Lichtung des Kopfhaares bezeichnet, welche im Stadium II und III kontinuierlich zunimmt. Die Abbildung zeigt eine Alopezie im Stadium II bis III.

AGA bei über $80 \%$ der Männer, während $20 \%$ auch eine AGA vom weiblichen Typ Female Pattern ausbilden können.

Weibliche Form. Die weibliche Form der AGA manifestiert sich als diffuse Lichtung, die im Oberkopfbereich unter Stehenlassen eines frontalen, ca. 1 bis $2 \mathrm{~cm}$ breiten Haarstreifens an der StirnHaar-Grenze beginnt. In weiteren Stadien, die 1977 von Ludwig klassifiziert worden sind, tritt eine zunehmende Intensität der diffusen Lichtung in der Oberkopfregion auf, ohne dass eine völlige Kahlheit resultiert [12] ( $\bullet$ Abb. 2).

Bei etwa $10 \%$ der Frauen ist die genetische Penetranz zur Entwicklung der AGA so stark, dass die Lichtung des Kopfhaares bereits im Alter zwischen 20 und 30 Jahren beginnt. Bei einigen Frauen resultiert mit der Umstellung der Hormonlage im Klimakterium ein Absinken der Östrogene verbunden mit einem relativen Androgenüberangebot, woraus sich eine AGA erst manifestiert oder - wenn vorbestehend - eine Verstärkung erfährt. Bei der Frau ist im Vergleich zum Mann die Aktivität der $5 \alpha$-Reduktase weniger relevant als die reduzierte Aktivität der Aromatase, die die anfallenden Androgene in nicht ausreichendem Maße in Östrogene umwandelt [9,13]. Als Konsequenz erfolgt wie beim Mann die Miniaturisierung der erblich prädisponierten Haarfollikel, wobei sich im Gegensatz zum Mann nie eine völlige Kahlheit der Kopfhaut entwickelt [14].

Pathogenese der AGA der Frau: Androgene und verminderte Aromatase-Aktivität

\section{Therapie der Alopecia androgenetica}

Für die Therapie der androgenetischen Alopezie stehen wenige, aber gut belegte therapeutische Optionen zur Verfügung.
Finasterid. Finasterid, ein $5 \alpha$-ReduktaseTyp2-Hemmer blockiert die intrafollikuläre enzymatische Umwandlung von Testosteron zu Dihydrotestosteron (DHT), welches das hauptverantwortliche Hormon für die Entwicklung der androgenetischen Alopezie in genetisch prädisponierten Haarfollikeln ist [15]. Die Wirkung von Finasterid wurde in einer placebokontrollierten, randomisierten Cross-over Studie an 1500 Männern mit AGA über insgesamt fünf Jahre untersucht. Dabei zeigte sich, dass bei $80 \%$ der behandelten Männer die Anwendung von $1 \mathrm{mg}$ Finasterid täglich zu einer signifikanten Zunahme der Haarzahl in definierten Arealen führte und auch in den Übersichtsfotografien eine Stabilität des Haarausfalls bzw. eine Verdichtung des Haares beobachtet werden konnte [16].

Cave: Finasterid ist kontraindiziert bei Frauen im gebärfähigen Alter!

Minoxidil. Minoxidil ist die zweite Substanz, für die eine Wirksamkeit bei der AGA belegt ist $[17,18]$. In einer placebokontrollierten, doppelblinden Multicenter-Studie mit 393 Männern (Alter: 18 - 49 J) mit AGA über 48 Wochen wurde für Minoxidil in der Konzentration von $5 \%$ ein signifikant früherer Wirkungseintritt und $45 \%$ stärkeres Haarwachstum gegenüber einer $2 \%$-igen Minoxidil-Lösung festgestellt [18]. Auch die Lebensqualität unter der $5 \%$-igen Lösung verbesserte sich, obwohl subjektive, direkte Nebenwirkungen wie Pruritus in der Gruppe mit 5\%-iger Lösung stärker ausgeprägt waren als in der Gruppe mit $2 \%$-iger Lösung [18]. Seit den frühen 90er-Jahren ist Minoxidil auch bei Frauen mit AGA untersucht worden. Bei 28 betroffenen Frauen (Alter: 22-44 J) zeigte sich über einen Behandlungszeitraum von 32 Wochen, dass sich in der Gruppe mit $2 \%$-iger Minoxidil-Lösung die Haarzahl signifikant gegenüber der Gruppe mit Placebo erhöhte und sich bei $60 \%$ eine minimale bis mäßige Haarwachstumsverbesserung einstellte, während dies nur für $46 \%$ der mit Placebo Behandelten zutraf [19]. In einer größeren Multicenter-Studie mit 346 Frauen in 10 Zentren in Europa konnte über einen Behandlungszeitraum von 32 Wochen in der 2\%-igen Minoxidil-Gruppe eine Zunahme der Haarzahl von 33 Haaren versus 19 Haaren in der Placebo-Gruppe festgestellt werden [17]. In einer Vergleichsstudie zur Wirksamkeit und Sicherheit von 2\%- und 5\%-iger Minoxidil-Lösung bei 381 Frauen (Alter: 18-49 J) mit AGA konnte über einen Behandlungszeitraum von 48 Wochen durch beide Konzentrationen gebenüber Placebo eine signifikante Zunahme der Terminalhaaranzahl verzeichnet werden [20], wohingegen Nebenwirkungen wie Pruritus, Trockenheit der Kopfhaut, Schuppung und faziale Hypertrichose etwas häufiger unter Behandlung mit $5 \%$-iger vs. $2 \%$-iger

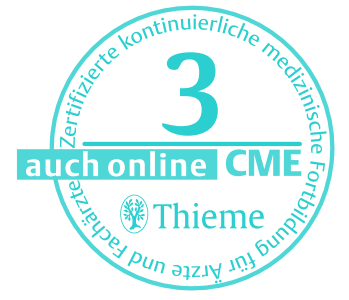


Minoxidil-Lösung festgestellt wurden. Nach Bewertung des Wirkung-Nebenwirkungsprofils beider Minoxidil-Konzentrationen hat sich daher für die Behandlung der AGA bei der Frau die $2 \%$-ige Konzentration als optimal erwiesen, was sich in der Zulassung dieser Konzentration seit 2004 in Deutschland widerspiegelt. In einer kürzlich publizierten Arbeit konnte auch für einen neu entwickelten Minoxidil-haltigen Schaum über 16 Wochen Behandlungsdauer eine signifikante Wirkung auf die Haaranzahl gegenüber Placebo gezeigt werden [21].

Finasterid und Minoxidil sind zur Behandlung der AGA des Mannes wirksame Therapiemöglichkeiten. Minoxidil in $2 \%$-iger Lösung ist für die Frau ein wirksames Therapieprinzip.

Weitere Therapieverfahren. Weitere Therapiemöglichkeiten sind die Anwendung von topischem $17 \alpha$-Estradiol sowohl bei der Frau als auch beim Mann, da diese synthetische $17 \alpha$-Form des Estradiols keine systemischen Wirkungen aufweist, bei topischer Applikation aber eine Hemmung der Dihydrotestosteron (DHT)Bildung bewirkt. Dies wurde zumindest in vitro an Rattenleberschnitten beobachtet und damit impliziert, dass $17 \alpha$-Estradiol die $5 \alpha$-Reduktase hemmt. Eine klinische Studie mit 96 Männern und Frauen zeigte durch Anwendung einer 0,025\%-igen $17 \alpha$-Estradiol-Lösung nach 12 Monaten Behandlung im Trichogramm eine Zunahme der Anagenrate um $10 \%$, während die Veränderung der Anagenrate in der Placebogruppe bei $4 \%$ lag. Für die Frau stehen zusätzlich topische Lösungen mit $17 \beta$-Estradiol zur Verfügung. Bei frühzeitig und/oder stark ausgeprägter AGA der Frau können auch Hormonpräparate mit verschieden stark gestaffelter antiandrogener Komponente verordnet werden wie z.B. Chlormadinonacetat, Dienogest und Cyproteronacetat. Bei gleichzeitig gewünschter kontrazeptiver Wirkung können für Frauen im gebärfähigen Alter v.a. Kombinationspräparate mit Chlormadinonacetat empfohlen werden. Diese sollten jedoch stets unter Rücksprache oder direkt vom Gynäkologen verordnet werden. Zur Behandlung der AGA des Mannes stehen im Stadium, in dem der Haarausfall zum Stillstand gekommen ist, operative Methoden zur Verfügung. Während Rotations- bzw. Verschiebeplastiken aufgrund der Größe und möglicher Komplikationen weitestgehend nicht mehr als Behandlungsmethode eingesetzt werden, stehen heute moderne Techniken der Mini- oder Mikro-Haartransplantation zur Verfügung. Die Mini- (3-5 Haare) oder Mikro(1-2 Haare) Transplantate (Grafts) werden aus dem androgen-resistenten okzipitalen Kopfhautbereich entnommen und in durch Mikro-Slit-
Skalpell- oder Laser-Technik vorbereitete Mikroschlitze im Temporal- und Vertexbereich eingesetzt.

\section{Alopecia areata}

Die Alopecia areata (AA; dt. kreisrunder Haarausfall) ist eine meist plötzlich auftretende Form eines umschriebenen Haarausfalls, der typischerweise im Anfangsstadium zunächst kreisrund ist und sich dann zentripetal oder multilokulär (AA multilocularis) ausbreiten kann. Ist nahezu das gesamte Kapillitium betroffen, spricht man von Alopecia areata subtotalis, bei komplettem Haarverlust des Kopfhaares von AA totalis. Außerhalb des Kapillitiums können auch Augenbrauen, Wimpern, Bartbehaarung bei Männern, Achsel und Pubesbehaarung betroffen sein. Sind sämtliche haartragenden Areale des Integumentes betroffen, liegt eine AA universalis vor. Als Begleitsymptome können Tüpfelnägel oder Querrillen der Nagelplatte beobachtet werden. Klinisch finden sich im Randbereich der Läsionen häufig sogenannte „Ausrufezeichenhaare“ (ca. 2-4 mm lang), die schmal bzw. depigmentiert aus der Kopfhaut austreten und nach distal hin breiter werden). Seltener sind dunkel pigmentierte Komedo-ähnliche Haarreste in betroffenen Follikelöffnungen zu sehen, die als „Kadaverhaare“ oder „points noirs“ bezeichnet werden. Die AA ist nach androgenetischer und diffuser Alopezie die dritthäufigste Form des Haarausfalls und kommt familiär gehäuft vor. Sie kann in jedem Lebensalter auftreten, hat aber eine gewisse Häufung zwischen dem 5. Lebensjahr und dem 3. Lebensjahrzehnt. Pathogenetisch wird am ehesten eine Autoimmungenese vermutet, da sich in Kopfhautbiopsien von Patienten mit AA in der Bulbusregion der Haarfollikel ein dichtes $\mathrm{CD}^{+}{ }^{+}$und $\mathrm{CD}^{+}$-Zellinfiltrat zeigt, deren Lymphozyten Zytokine wie IFN $\gamma$, IL-1b und IL-2 sezernieren, welche typisch für eine $\mathrm{TH}-1$ getriggerte Immunantwort sind und auf eine zellulär vermittelte Autoimmunreaktion hindeuten [13-22].

Verlauf. Der Verlauf und die Dynamik der AA ist extrem mannigfaltig und kann von einem einmal auftretenden kleinen Herd mit Spontanremission über multiple größere Herde und ein Nebeneinander von sich bessernden Herden neben an anderer Stelle neu auftretenden Herden bis zu einer über viele Jahre bestehenden Haarlosigkeit gehen, die dann nahezu irreversibel wird (๑ Abb. 3). Die Alopezia areata ist jedoch prinzipiell eine reversible Erkrankung. Häufig treten die Herde parietal oder okzipital auf und können im Okzipitalbereich häufig als streifiges haarloses Areal imponieren (Ophiasis-Typ). Bei einem Drittel der Patienten tritt nach Erstmanifestion im Verlauf von 6 Monaten eine Spontanremission ein und nahezu die Hälfte der Patienten sind nach einem Jahr erscheinungsfrei [13]. 

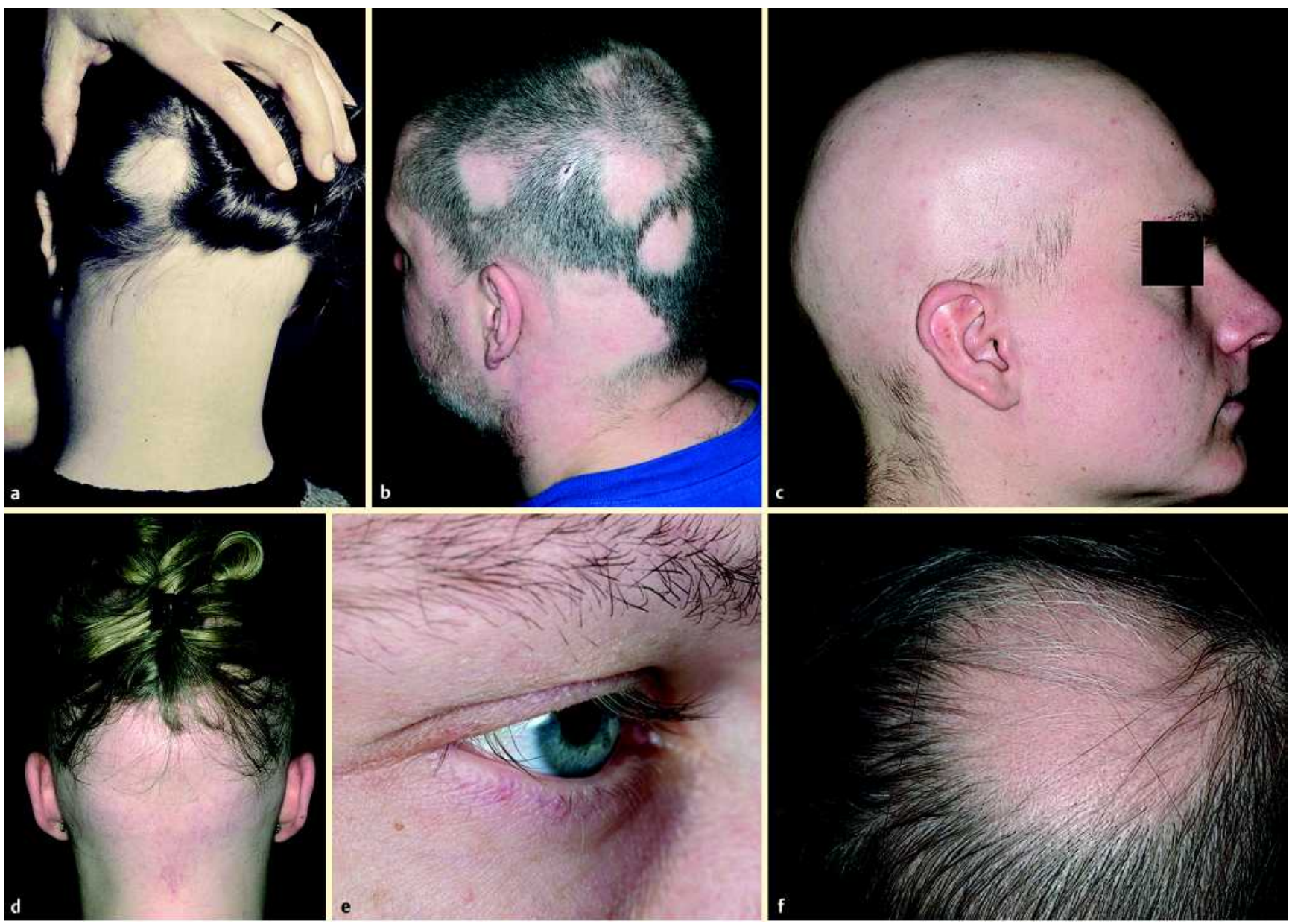

Abb. 3 Verschiedene Formen und Manifestationen der Alopecia areata: unilocularis pie mit Kortikosteroiden der Wirkklasse III und IV (z.B. Mometason, Amcinonid; Clobetasol17-Propionat) je nach Akuität und Befall über 4-6 Wochen angewandt werden (Evidenzlevel III [22]). In einer relativ neuen Studie zur Anwendung von Clobetasol-Schaum über 12 Wochen konnte im Halbseitenversuch bei $89 \%$ der Behandelten ein Haarwiederwachstum von $\geq 25 \%$ gegenüber $11 \%$ auf den placebobehandelten Feldern beobachtet werden [23]. Alternativ kann bei wenigen Herden (z.B. 4-6) eine intraläsionale Injektion von Triamcinolon-Kristallen mittels Kanüle oder Dermo-Jet versucht werden. Die (a); multilocularis (b); subtotalis (c); Ophiasis-Typ (d); Befall der Wimpern (e); nachwachsende depigmentierte Haare bei Alopecia areata in Remission (f). her trotz relativ guter Evidenzlage (III) mittlerweile im eigenen Haus zurückhaltend praktiziert. Systemische Kortikosteroide. Bei zögerlichem Ansprechen bzw. ausgeprägter Dynamik im prolongiert-perakuten Verlauf ist die Therapie mit systemischen Kortikosteroiden zu empfehlen. Unter einer mittelhohen Dosierung von initial $50 \mathrm{mg}$ Prednisolon absteigend auf $10 \mathrm{mg}$ über 4 Wochen (siehe $\bullet$ Tab. 1) kann nicht selten ein Sistieren des Haarausfalls (negativer Pull-Test) mit Remissionstendenz erreicht werden. Bei An-

Tab. 1 Behandlungs-Schema der oralen KortikosteroidTherapie über 4 Wochen zur Behandlung eines akuten Schubes der Alopecia areata

\begin{tabular}{|l|l|}
\hline Dosis & Dauer \\
\hline $50 \mathrm{mg}(2 \times 20 \mathrm{mg}+2 \times 5 \mathrm{mg})$ & 3 Tage $(\operatorname{Tag} 1-3)$ \\
\hline $40 \mathrm{mg}(2 \times 20 \mathrm{mg})$ & 3 Tage $(\operatorname{Tag} 4-6)$ \\
\hline $30 \mathrm{mg}(1 \times 20 \mathrm{mg}+2 \times 5 \mathrm{mg})$ & 3 Tage $(\operatorname{Tag} 7-9)$ \\
\hline $25 \mathrm{mg}(1 \times 20 \mathrm{mg}+1 \times 5 \mathrm{mg})$ & 5 Tage $(\operatorname{Tag} 10-14)$ \\
\hline $20 \mathrm{mg}(=1 \times 20 \mathrm{mg})$ & 5 Tage $(\operatorname{Tag} 15-19)$ \\
\hline $15 \mathrm{mg}(=3 \times 5 \mathrm{mg})$ & 5 Tage $(\operatorname{Tag} 20-24)$ \\
\hline $10 \mathrm{mg}(=2 \times 5 \mathrm{mg})$ & 5 Tage $(\operatorname{Tag} 25-29)$ \\
\hline
\end{tabular}

Therapie ist jedoch relativ schmerzhaft, verlangt häufige Visitentermine und ist im Bereich frontal der Transversallinie kontraindiziert (Emboliegefahr der A. retinalis od. nervi optici) und wird da-

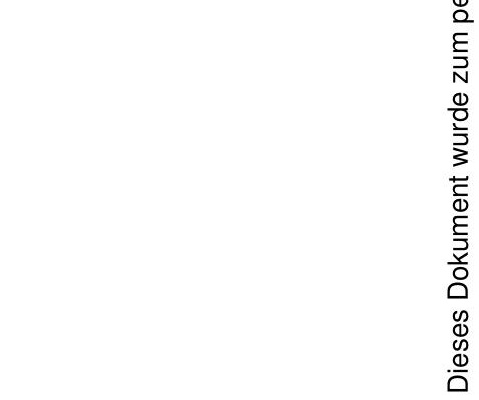


sprechen kann noch einmal über 4 Wochen verlängert werden ( 2 Wo $20 \mathrm{mg}, 2$ Wo $10 \mathrm{mg}$ ). Eine längere Therapiedauer ist unter Berücksichtigung der dann zunehmenden Nebenwirkungsrate und der gleichzeitig unwahrscheinlicher werdenden Erfolgschance nicht zu empfehlen.

Puls- bzw. Intervalltherapien mit hochdosierten Steroiden (z.B. 400-500 mg Methylprednisolon i.v. für 3 Tage oder $5 \mathrm{mg} / \mathrm{kg} \mathrm{KG}$ oral einmal monatlich für 3-9 Monate) zeigten bei einigen Autoren und eigenen Untersuchungen zwar partielles Ansprechen, jedoch selten eine dauerhaft vollständige Remission in kontrollierten Studien. Pathophysiologisch ist in allen Fällen der Steroidtherapie am ehesten von einem Durchbrechen der Dynamik der entzündlichen Reaktion am Haarfollikel auszugehen, welche eine vorzeitige Spontanremission einleitet.

Topische Immuntherapie. In allen Fällen, in denen dieses Vorgehen über eine gewisse Zeit erfolglos ist und ein alopezisches Areal von $>30 \%$ besteht, steht die topische Immuntherapie mit Diphenylcyclopropenon (DCP) und Squaric-Acid-Dibutylester (SADBE; Quadratsäure-Dibutylester) als Therapieoption mit guter Evidenzlage (Level II) zur Verfügung. DCP und SADBE stellen ein in der üblichen Umwelt nicht vorkommendes chemisches obligates Allergen dar, welches initial auf ein umschriebenes Areal der Kopfhaut in 2\% Aceton-Lösung eine Sensibilisierung auslöst. Zwei Wochen nach Sensibilisierung wird in kleinsten Konzentrationen wöchentlich ansteigend (Beginn 0,0001\%) ein allergisches Ekzem ausgelöst, welches mit Erythem, Pruritus und ggf. Lymphknotenschwellung für 2-3 Tage in einem für den Patienten tolerablen Maß einhergehen soll. Hat sich das Ekzem reproduzierbar eingestellt, kann in 1-6 Monaten mit einem Wiederwachstum bis zur Vollremission gerechnet werden. Die Ansprechrate liegt je nach klinischem Ausgangsbefund bei $29-78 \%$ (median 49\%) (detaillierte Übersicht hierzu in [22]).

Weitere Therapieverfahren. Für weitere Therapieverfahren wie die topische Anwendung von Dithranol fehlen klinisch kontrollierte Studien. Auch die PUVA-Turban-Therapie zeigt zwar über 16 Wochen ein Ansprechen, ist aber durch eine Rezidivrate von 30-50\% gekennzeichnet. Bei Anwendung des 308-nm-Excimer-Laser in 24-27 Sitzungen über 3 Monate sind bei der patchy AA erfolgreiche Verläufe beschrieben, jedoch nicht in kontrollierten Studien belegt. Auch die bzgl. der Autoimmunpathogenese vermuteten Therapieansätze mit Biologics (z. B. TNF $\alpha$-Blocker Etanercept oder Infliximab) haben sich bisher als erfolglos erwiesen. Die Anwendung von Zink als Immunmodulator (z. B. Unizink ${ }^{\circledR} 50 \mathrm{mg} 2 \times /$ die) kann aufgrund des günstigen Nebenwirkungsprofils und teilweise klinisch empirischen Erfolgen erwogen werden. Die Evidenzlage hierfür ist jedoch nicht sehr stark.
Topische und systemische Kortikosteroide sind zur Induktion der Spontanremission einsetzbar. DCP ist die einzige evidenzbasierte Therapie (EvidenzLevel II).

\section{Diffuse Alopezie}

Diffuse Alopezien umfassen Formen von Haarausfall, denen allen ein diffus über das gesamte Kapillitium verteilter Haarausfall zugrunde liegt und sich daher im klinischen Bild von der Alopecia androgenetica, der Alopecia areata und umschriebenen vernarbenden Alopezien unterscheiden. Häufig liegt einer diffusen Alopezie eine vorübergehende systemische Veränderung zugrunde, weswegen sie auch als symptomatische Alopezie bezeichnet wird ( $\odot$ Abb.4). Zur Inzidenz und Prävalenz der diffusen Alopezien gibt es keine aussagekräftigen Untersuchungen, jedoch zum relativen Risiko in Abhängigkeit der verschiedenen Auslösefaktoren. Pathoätiogenetisch liegt der diffusen Alopezie entweder eine absolute oder relative Mangelsituation am Haarfollikel oder die Überpräsenz von toxischen Substanzen zugrunde. Die nutritive Relevanz bzw. toxische Potenz, Dosis und Applikationshäufigkeit hat direkten Einfluss auf die Ausprägung und die Entwicklungsdynamik der diffusen Alopezien.

Einteilung. Es wird das Effluvium vom dystrophischen oder Frühtyp (Dystrophes Effluvium) und das Effluvium vom verzögerten oder Spättyp (Telogeneffluvium) unterschieden [24-26]. Im ersten Fall wird das Haarwachstum in der Anagenphase abrupt abgebrochen, sodass die Katagen- und Telogenphase nicht mehr durchlaufen wird und es zu einem rasch einsetzenden Haarausfall (Tage bis Wochen) von überwiegend dystrophen Anagenhaaren kommt. Beim Spättyp wird die Anagenphase der Haarfollikel durch die Schädigung zeitlich synchron beendet und alle betroffenen Haare gleichzeitig in das Katagenund Telogenstadium getrieben, wodurch klinisch ein perakuter Haarausfall (3-6 Monate) resultiert. Auch wenn die klinische Manifestation nicht selten stark und für den Patienten schockierend ist, stellt die in vielen Fällen vorhandene volle Reversibilität für die betroffenen Patienten eine positive Tatsache dar, die der trichologisch kompetente Arzt in der Patientenführung unbedingt klar herausstellen sollte.

Ursachen. Mögliche Ursachen für diffuse Alopezien sind beispielsweise Stoffwechsel- und hormonelle Erkrankungen (Diabetes mellitus, Hypound Hyperthyreose, Prolaktinom), Eisenmangel, Vitaminmangel, Proteinmangel, schwere mit Fieber einhergehende systemische Entzündungen (z. B. Pneumonie), Vollnarkose, Operation, systemischer Lupus erythematodes, Lues (frühes Stadium II), chronisch-entzündliche Darmerkrankungen oder auch Malignome wie zum Beispiel 


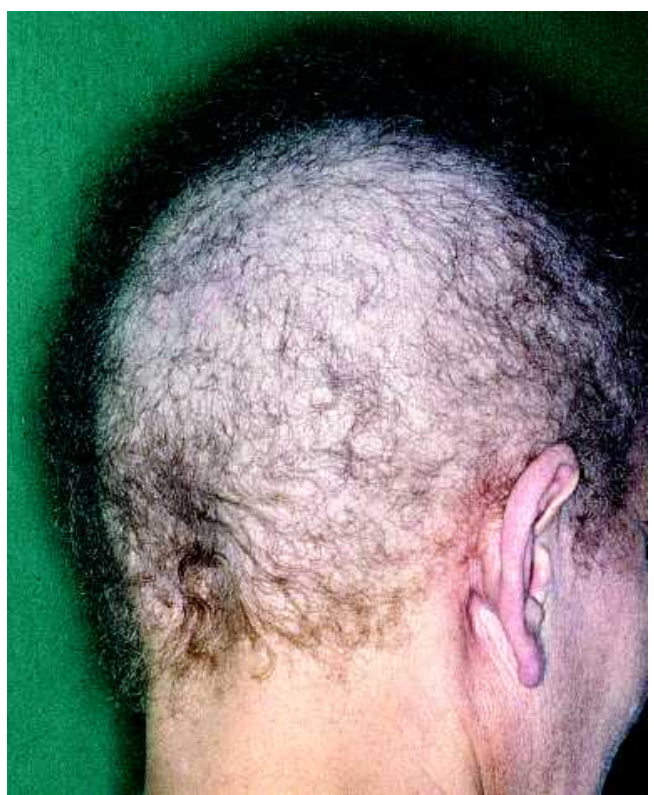

Abb. 4 Klinisches Bild der diffusen Alopezie bei einem Mädchen nach schwerem grippalem Infekt mit sekundärer bakterieller Pneumonie.

maligne Lymphome (Alopecia muzinosa). Auch die bei einigen Frauen zu beobachtende Alopecia post partum ist eine diffuse Alopezie, ausgelöst durch einen akuten relativen Östrogenmangel. Eine Vielzahl von Medikamenten kann ebenfalls eine diffuse Alopezie auslösen wie z.B. ACEHemmer, Betablocker, Retinoide, Thyreostatika aber auch L-Thyroxin, Antibiotika, Analgetika und v.a. chemotherapeutische Substanzen (Interferon selten; häufiger Carboplatin (2\%), Etoposid (66\%), Ifosfamid (bis 80\%) und Doxorubicin (knapp 100\%) [27].

Bei der akuten AD kommt es zu dystrophem Anagen-Effluvium, bei der perakuten AD zum Telogeneffluvium.

\section{Therapie der Alopecia diffusa}

Die kausale Therapie bei diffuser Alopezie besteht aus der Erkennung und Elimination der auslösenden Noxe/systemischen Erkrankung bzw. des verursachenden Mangelzustandes. Die Aufklärung über die Reversibilität ist Hauptelement des therapeutischen Gespräches. Bei chemotherapieinduziertem Haarausfall ist eine Verkürzung der Latenzperiode bis zum Wiederwachstum des Haares durch Minoxidil beschrieben worden.

\section{Vernarbende Alopezien}

Vernarbende Alopezien stellen eine pathogenetisch sehr inhomogene Krankheitsgruppe dar, die aber klinisch alle eine gemeinsame Endstrecke erreicht haben oder potenziell im Verlauf der Erkrankung erreichen können: eine Vernarbung der Haarfollikel mit dauerhaftem Haarverlust (irreversible Alopezie). Histomorphologisch und funktionell liegt dieser eine Zerstörung des Wurzelorgans (dermale Papille) und/oder der Wulstregion („,bulge“; Ansatzpunkt des M. arrector pili; Sitz der Stammzellen) zugrunde. Die pathologischen Ursachen sind entzündlich, mechanisch-physikalisch (z. B. posttraumatisch, Radioderm) oder im Rahmen von Kollagenosen. Während eine oberflächliche bakterielle oder mykotische Entzündung der Kopfhaut keine vernarbende Alopezie induziert, können tiefe Pyodermien/Mykosen/Trichophytien, die das Wurzelorgan und die Wulstregion betreffen, zu irreversiblem Haarverlust führen. Im Folgenden werden die häufigsten Formen der vernarbenden Alopezien, die insgesamt im Vergleich zur AGA und $A A$ jedoch relativ selten sind, beschrieben.

\section{Lichen ruber follicularis}

Der Lichen ruber des behaarten Kopfes (auch Lichen planopilaris) manifestiert sich in fleckartigen entzündlichen Alopezie-Herden, in denen die Haarfollikel von perifollikulären Erythemen und Hyperkeratosen unterschiedlicher Ausprägung umgeben sind und sich im Laufe der Erkrankung eine Vernarbung entwickeln kann. Histologisch zeigt sich unterhalb der Basalmembranzone eine bandförmige Ansammlung von überwiegend $\mathrm{CD}^{+}{ }^{+}$-T-Lmyphozyten neben vereinzelten Histiozyten. Ätiopathogenetisch wird eine abnormale Immunantwort auf ein unbekanntes Antigen der Basalmembranhone vermutet. Finden sich am übrigen Integument weitere Symptome eines Lichen ruber wie Wickham'sche Streifung der Mundschleimhaut, follikuläre oder polygonale Papeln der Felderhaut und Nageldystrophien wird dieses Bild als Lasseur-GrahamLittle-Syndrom bezeichnet $[26,28]$. Zur Diagnostik und Abgrenzung zu anderen vernarbenden Alopezien sollte eine Spindelbiopsie, die sowohl vernarbende als auch haartragende, erythematös-entzündliche Areale erfasst, angestrebt werden. Neben dem bandförmigen lymphozytären Infiltrat in der HE-Färbung können mittels direkter Immunfluoreszenz unspezifisch fluoreszierende Kolloidkörperchen (sog. Cytoid-Bodies) und vaskuläre Fibrinablagerungen nachgewiesen werden, wodurch histologisch eine Abgrenzung vom chronisch-diskoiden Lupus erythematodes vorgenommen werden kann [13].

Kombination von Lichen ruber follicularis und polygonale oder follikulär gebundene Papeln am Integument und Nagelveränderungen deuten auf ein Lasseur-Graham-Little-Syndrom hin.

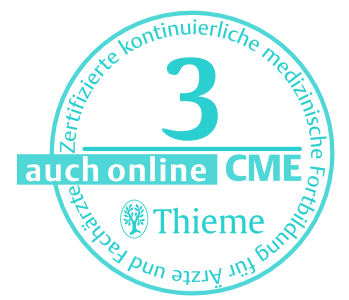



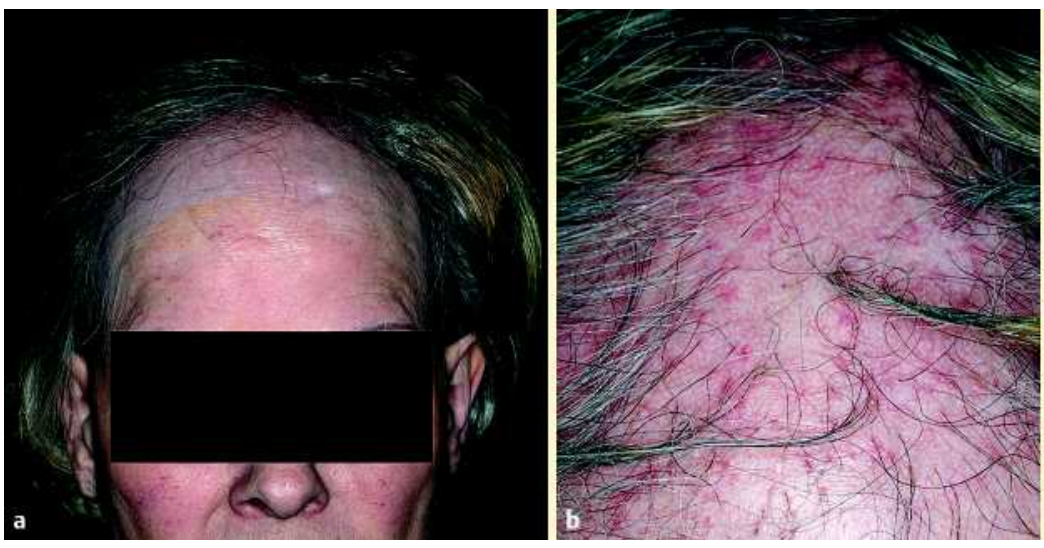

Abb. 5 Postmenopausale frontal-fibrosierende Alopezie Kossard: frontale Ansicht (a) Detailaufnahme mit perfollikulären Erythemen und Hyperkeratosen (b); Typischer Befall der temporo-frontalen Haarpartien (c).

Therapie. Therapeutisch ist der Lichen ruber follicularis schwierig unter Kontrolle zu halten, da er durch eine relativ therapieresistente langsame Progredienz der Vernarbung über Jahre gekennzeichnet ist. Trotzdem sollte im entzündlichen Stadium stets eine der Krankheitsdynamik entsprechende anti-inflammatorische Therapie durch Kortikosteroide erfolgen. Diese kann von topischen Steroiden verschiedener Wirkstärke über lokale Injektionen von Triamcinolon-Kristallsuspension bis zu oral-systemischen Steroiden bis zu i. v.-applizierten Hochdosis-Stoßtherapien (z.B. $100 \mathrm{mg}$ Fortecortin an 3 aufeinanderfolgenden Tagen in 4-wöchigem Abstand) reichen.

Cave: Frontal der Transversallinie des Kopfes, können Embolien der A. retinalis oder nervi optici ausgelöst werden.

Parallel kann die Therapie mit Acitretin (ca. $0,5 \mathrm{mg} / \mathrm{kg} \mathrm{KG}$ ) versucht werden. Eine komplette Remission ist selten zu erreichen. Daher ist das Ziel der Behandlung, ein Sistieren der fortschreitenden Vernarbung zu erreichen. Parallel sollte mit dem Patienten eine kosmetische Lösung der Alopezie erörtert werden (professionell angeschweißte Haarsträhnen, Haarteil, Perücke). Im sog. „ausgebrannten“ Zustand kann eine Haartransplantation erwogen werden.

Ziel der Therapie mit topischen oder systemischen Kortikosteroiden sollte ein Sistieren der Entzündungsreaktion und des Fortschreitens der Vernarbung sein. Nach erfolgloser Therapie mit Kortikosteroiden (topisch bis systemisch hochdosiert) und Acitretin können kosmetische Maßnahmen und im ausgebrannten Zustand eine Haartransplantation erwogen werden.

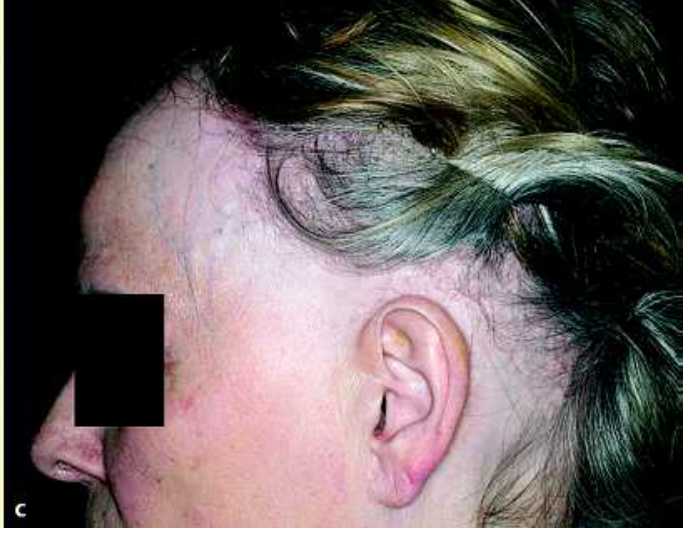

Postmenopausale frontal-fibrosierende Alopezie Kossard

Das Zurückweichen der frontalen oder frontotemporalen Haarlinie bei postmenopausalen Frauen einhergehend mit perfollikulären Erythemen und Hyperkeratosen ist als postmenopausale frontal-fibrosierende Alopezie (PFFA) von Kossard 1994 erstmals beschrieben worden (৫ Abb. 5) [29]. Histologisch zeigt sich ein bandförmiges lymphozytäres Infiltrat um die oberen Anteile des Haares (Isthmus, Infundibulum), welches sich vom Lichen planopilaris nicht unterscheidet und ebenfalls zu sukzessiver Vernarbung führen kann [30]. Klinisch lassen sich beide Diagnosen unterscheiden: Während der Lichen planopilaris fleckartig an allen Stellen des Kapillitiums auftreten kann, findet sich die PFFA nur im frontalen Bereich mit Präferenz der inflammatorischen Reaktion an androgen-sensitiven Intermediär- und Vellushaarfollikeln. Aufgrund der ähnlichen klinisch-histologischen Morphologie wird die PFFA jedoch auch als Spielart des Lichen planopilars gesehen [31]. Häufig besteht auch eine Ko-Ätiopathogenese mit der postmenopausalen androgenetischen Alopezie der Frau $[30,32,33]$. Zusätzlich können die Augenbrauen partiell oder komplett in 50-65\% und die Achseln in 37\% der Fälle von PFFA betroffen sein. Die partielle androgenetische Assoziation spiegelt sich bei etwa $40 \%$ der Fälle im parallel bestehenden klinischen Erscheinungsbild einer androgenetischen Alopezie und in der therapeutischen Ansprechbarkeit auf Finasterid und Antiandrogene, welche von einigen Autoren berichtet wird, wider [30,32].

Therapie. Neben der beschriebenen Therapie mit Finasterid und Antiandrogenen kann alternativ die Behandlung mit topischen oder systemischen mittel- oder hochdosierten Kortikosteroiden partielle Erfolge erzielen. Lässt sich die Entwicklung der Erkrankung nicht frühzeitig aufhalten, resultiert eine vernarbende Alopezie, die im Maximalstadium mit einer flächenhaften Ausprägung als „Clown-Alopezie“ imponieren kann [30], welchen mit haarkosmetischen Maßnahmen (Haarteil, Perücke) begegnet wird. 
Lupus erythematodes capillitii

Der Lupus erythematodes capillitii ist eine am Kapillitium lokalisierte Form des chronisch diskoiden Lupus erythematodes (CDLE), der klinisch und histologisch mit dem CDLE des Integumentes identisch ist. In unregelmäßiger Verteilung, jedoch häufig im Oberkopf-/Vertexareal, finden sich fleckartige entzündlich-erythematöse, teils livide Plaques, die zentral eine glatt-glänzende porzellanartige Atrophie entwickeln [13,26,34]. Charakteristischerweise findet sich eine randständige Schuppung sowie an vernarbenden Follikelostien oder an noch verbleibenden Haarfollikeln lokalisierte perifollikuläre Hyperkeratosen, die sich kegelartig aushebeln lassen („Tapeziernagelphänomen“) ( $\bullet$ Abb. 6). Histologisch findet sich eine vakuolige Degeneration der basalen Epidermis und ein meist scharf begrenztes fleckförmiges lympho-histiozytäres in das tiefere Korium absteigendes Infiltrat, welches perivaskulär und -follikulär entwickelt ist. Parallel finden sich follikuläre Hyperkeratosen und eine Verquellung und Verbreiterung der Basalmembranzone in der PAS-Färbung. Der autoimmunen Pathogenese entsprechend lassen sich in der direkten Immunfluoreszenz granuläre Ablagerungen von IgG und C3 in der dermo-epidermalen Junktionszone nachweisen. Im meist chronischen Verlauf der Erkrankung gehen die entzündlichen Herde in vernarbende Plaques über, die im „ausgebrannten" Stadium in den État pseudopeladique münden $[35,36]$. Diagnostisch finden sich nur bei $20 \%$ der Patienten antinukleäre Antikörper [13].

Therapie. Therapeutisches Ziel ist, das Fortschreiten der vernarbenden Alopezie zu verhindern. Hierbei kommen in der ersten Stufe topische Kortikosteroide der Wirkklassen III und IV zum Einsatz, auch unter Okklusion und v.a. in den entzündlichen Randbereichen. Ist über 3-4 Wochen kein Rückgang des entzündlichen Erythems festzustellen, kann auf eine systemische Therapie mit Chloroquin bzw. Hydroxychloroquin (3,5-4,0 mg/KG Idealgewicht bzw. 6,0-6,5 mg/ $\mathrm{kg}$ Idealgewicht) gewechselt werden. Augenärztliche Untersuchungen zur Früherkennung einer potenziell auftretenden Retinopathie sollten bei Beginn, nach 3 Monaten und dann halbjährlich durchgeführt werden. Alternativ wird auch Dapson mit initial $100 \mathrm{mg} /$ die eingesetzt $[13,26]$. Prinzipiell ist den Patienten konsequenter Sonnenschutz zu empfehlen (Kopfbedeckung, Sunblocker mit hohem SF) - einerseits zur Reduktion der CDLE-Herdentwicklung, andererseits aber auch zur Prophylaxe der Karzinomentstehung in den durch Vernarbung und Haarlosigkeit schutzlos dem Sonnenlicht ausgesetzten Herden. Die Prognose ist v.a. bei Frauen in jüngerem Alter schlecht. Im über längere Zeit bestehenden entzündungsfreien État pseudopeladique kann eine Eigenhaartransplantation erwogen werden.
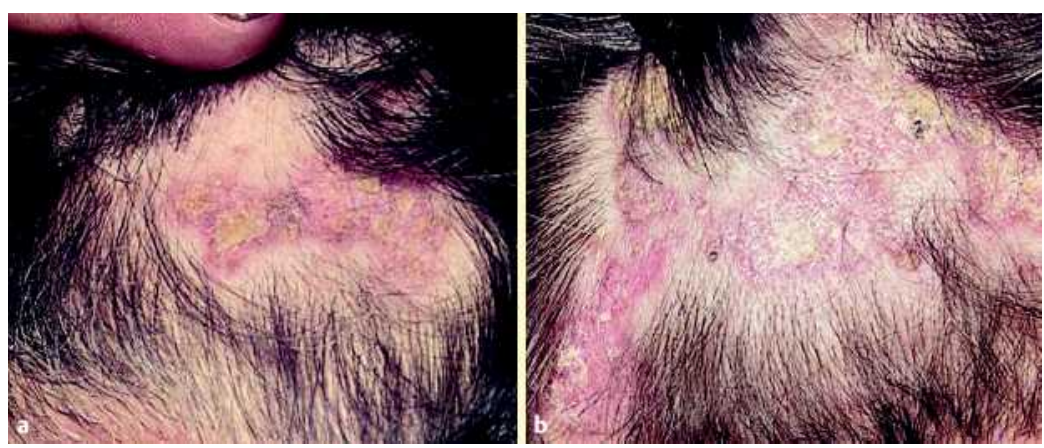

Abb. 6 Lupus erythematodes capillitii im akut-entzündlichen Stadium (a) und im ,ausgebrannt" vernarbenden Stadium (b).

Nach therapierefraktärem Einsatz von Kortikosteroiden Wirkklasse III/IV ist Chloroquin bzw. Hydroxychloroquin Mittel der 1. Wahl, alternativ kann Dapson eingesetzt werden.

\section{Folliculitis decalvans}

Regelmäßig finden sich bei dieser vernarbenden Alopezie Pusteln mit einer granulozytär geprägten Entzündung der Kopfhaut, die mit seröser Krustenbildung und Destruktion der betroffenen Haarfollikel einhergeht. Mikrobiologisch lässt sich aus den Pusteln meist Staphylococcus aureus nachweisen und histologisch kann eine granulozytär-destruierende Entzündung des Haarfollikels festgestellt werden [13]. Die Immunfluoreszenz ist negativ. Obwohl die betroffenen Patienten eine offensichtlich gestörte Abwehrreaktion gegen sonst harmlose Keime der physiologischen Hautflora entwickeln, ist bislang kein definierter immunologischer oder struktureller Defekt der Haarfollikel bzw. der interfollikulären Kopfhaut identifiziert worden.

Therapie. Therapeutisch sollte eine systemische Antibiotikatherapie nach Antibiogramm über 2-4 Wochen durchgeführt werden und parallel immunsuppressiv mit Kortikosteroiden (z.B. Prednisolon) $1 \mathrm{mg} / \mathrm{kg} \mathrm{KG}$ behandelt werden. Bei leichteren Formen kann auch zunächst nur eine topische Therapie mit einer Antibiotikum/Steroid-Kombination (z.B. Betametason-17-Valerat und Fusidinsäure) angewandt werden. Als Heilversuch kann auch eine Therapie mit Dapson (75 oder $100 \mathrm{mg}$ initial; $25 \mathrm{mg}$ Erhaltungsdosis) über mehrere Monate bis Jahre durchgeführt werden [37]. Bei einzelnen Patienten wurde eine über ein Jahr anhaltende Remission unter der Therapie mit topischer Fusidinsäure und oralem Zinksulfat (z. B. Solvezink ${ }^{\circledR} 3 \times 200 \mathrm{mg} /$ die; Unizink $^{\circledR}$ $2 \times 50 \mathrm{mg} /$ die) beobachtet [38]. Relativ überzeu-

Die Therapie erfolgt mit Antibiotika, Kortikosteroiden, Dapson. Gute therapeutische Erfolge erzielt man durch Kombinationstherapie mit Clindamycin $2 \times 300 \mathrm{mg} /$ die und Rifampicin $2 \times 300 \mathrm{mg} /$ die.

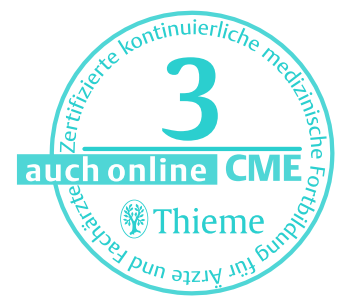



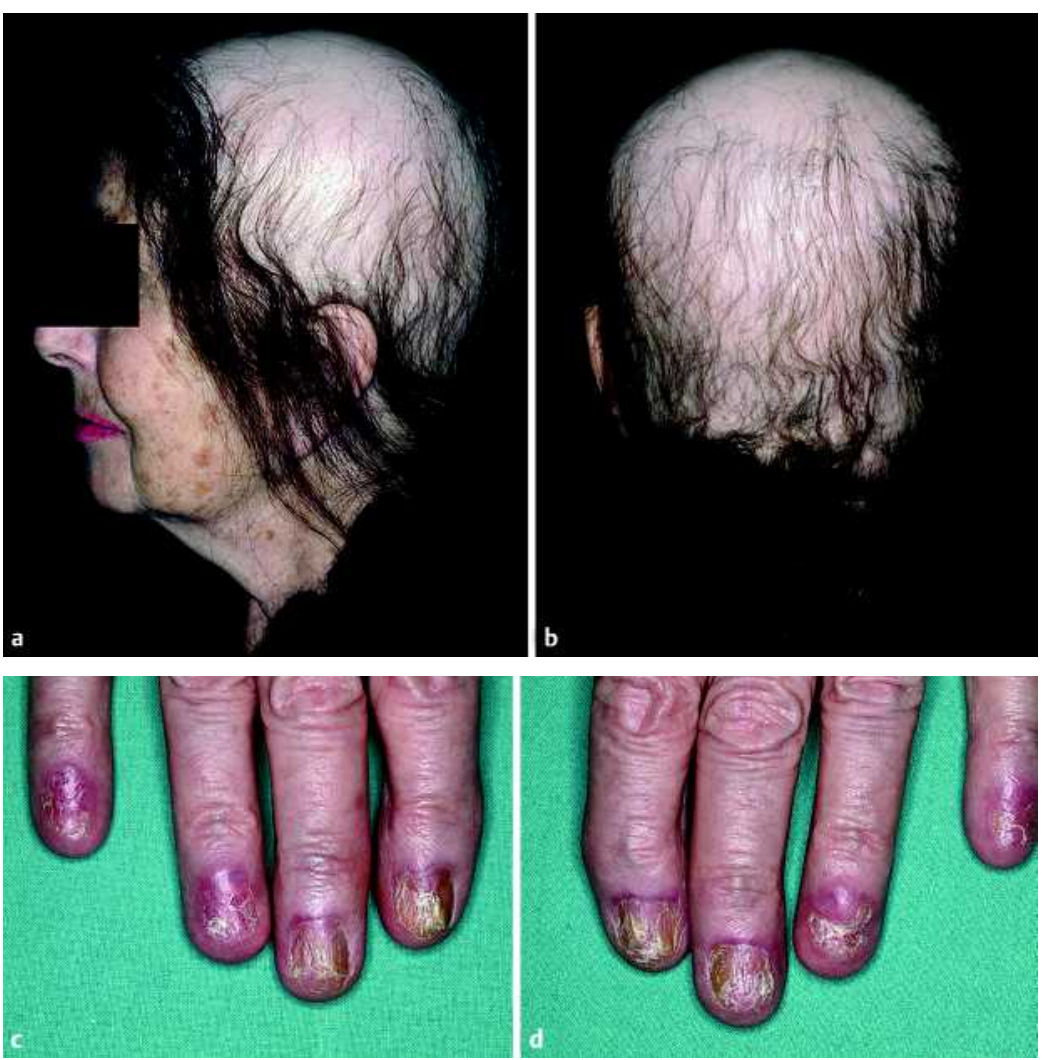

Abb. 7 État pseudopeladique bei Z.n. Lichen ruber capillitii im Rahmen eines Lasseur-Graham-Little-Syndroms. Subtotale Alopezie mit porzellan-glänzender Vernarbung unter Belassung eines frontalen Haarstreifens (a); nahezu komplette Alopezie im Okzipitalbereich (b). Dystrophe Fingernägel an beiden Händen (c), (d).

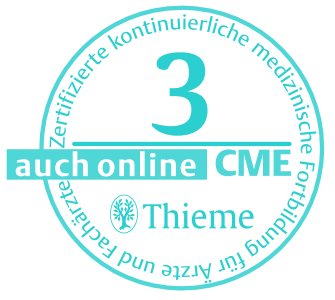

gende Remissionen über Monate bis Jahre konnten bei 75 bis $80 \%$ der Patienten unter der Kombinationstherapie von Clindamycin $2 \times 300 \mathrm{mg} /$ die und Rifampicin $2 \times 300 \mathrm{mg}$ bei insgesamt 26 Patienten über 10 Wochen Therapiedauer erzielt werden $[39,40]$.

\section{Pseudopelade Brocq und État pseudo- peladique}

Die Pseudopelade Brocq (PPB) ist ein von Brocq 1888 beschriebenes Krankheitsbild, dessen eigenständige Entität immer wieder angezweifelt wird [13,26,36,41]. Das Krankheitsbild ist durch fleckartige, narbig-fibrotische Herde mit sekundärer Destruktion der Haarfollikel charakterisiert und unterscheidet sich klinisch im Vergleich zum diskoiden Lupus erythematodes capillitii (CDLE) und Lichen ruber follicularis (LRF) v.a. durch ein deutlich geringer ausgeprägtes Entzündungserythem, welches auch histologisch einem geringen Entzündungsinfiltrat entspricht $[13,36]$. Im sekundär vernarbten Endzustand von CDLE, LRF oder Folliculitis decalvans spricht man vom État pseudopeladique (EPP) ( $\bullet$ Abb. 7), bei dem die Unterscheidung zur PPB sich häufig schwierig gestaltet. Eine umfangreiche Untersuchung von Braun-Falco et al. an 142 Erkrankten zeigte aber, dass selbst 20 Jahre nach Erstmanifestation bei CDLE und LRF teilweise noch histologische Merkmale dieser Erkrankungen (Pigmentinkontinenz, vakuolige Basalzelldegeneration) zu diagnostizieren waren, während bei $35 \%$ der Patienten auch im 1. Jahr der Erkrankung keine andere Diagnose als eine PPB zu stellen war [36]. Auch wenn die eigenständige Entität der PPB vielfach angezweifelt wird, kann sie als Begriff für jede vernarbende Alopezie verwendet werden, bei der anamnestisch, klinisch oder histologisch keine spezifische Dermatose des Kapillitiums klassifiziert werden kann, während EPP eine Phänokopie der PPB bei sekundärem Endzustand einer klassifizierbaren vernarbenden Alopezie darstellt.

Therapie. Therapeutisch ist das Ziel, die Ausbreitung der erkrankten Fläche zu verhindern, wobei topische Kortikosteroide (auch intraläsional) zur Anwendung kommen. Einige Erfolge sind auch unter der Behandlung mit Dapson $100 \mathrm{mg} /$ die für 4-6 Wochen beschrieben worden [26].

PPB wird als Begriff bei vernarbender Alopezie ohne detektierbare spezifische Diagnose verwendet. EPP ist der Zustand der Vernarbung nach Ablauf einer spezifischen Diagnose.

\section{Sklerodermie}

Die Sklerodermie kann eine umschriebene vernarbende Alopezie bedingen, wenn sie, typischerweise, als „En coup de sabre“-Sklerodermie auftritt. Bei dieser Variante der zirkumskripten Sklerodermie findet sich meist frontotemporal eine von kranial nach kaudal reichende longitudinale tiefe Atrophie der Kopfschwarte, die mit einem kompletten Verlust der Haarfollikel einhergeht.

Therapie. Hierzu ist keine kausale Therapie bekannt. Über die therapeutische Ansprechbarkeit von Penicillin, die für die zirkumskripte Sklerodermie des Integumentes beschrieben ist, gibt es bislang keine Angaben. Somit kommen symptomatische Prinzipien wie lokal abdeckende Maßnahmen wie Einarbeiten eines Haarteils oder Eigenhaartransplantation zum Einsatz.

\section{Sonstige Haarerkrankungen Loses-Anagen-Haar-Syndrom}

Ursache dieser seltenen Haarerkrankung ist eine gestörte vorzeitige Keratinisierung der Huxley'und Henle'schen-Schicht der inneren Wurzelscheide. Daraus resultiert eine Diskontinuität und mangelnde Anlagerung der inneren Wurzelscheide an den Haarschaft, wodurch es zu einem sehr lockeren Sitz des Haares in der Wurzelscheide und folglich in der Kopfhaut kommt $[42,43]$. Klinisch manifestiert sich dieses Krankheitsbild in sehr leicht (schmerzlos) bereits durch geringen Zug epilierbaren Haaren, v.a. in mechanisch etwas stärker beanspruchten Regionen des Kapillitiums (okzipital) v. a. bei blonden Kindern, teil- 
weise familiär gehäuft [44]. Die epilierten Haare sind ausschließlich dystrophe Anagenhaare, was im Trichogramm als wesentliches diagnostisches Kriterium etabliert ist. Histologisch zeigt sich eine Spaltbildung zwischen den Haarschäften und der inneren Wurzelscheide.

Therapie. Eine spezifische Therapie ist nicht bekannt, und betroffenen Patienten kann lediglich empfohlen werden, Traumen und Zugkräfte zu vermeiden. Eine Besserung des Krankheitsbildes mit zunehmendem Lebensalter wird beobachtet.

Beim Losen-Anagen-Haar-Syndrom kommt es zu einer Störung der Verankerung zwischen Haarschaft und innerer Wurzelscheide. Der Pull-Test ist positiv.

\section{Short-Anagen-Syndrom}

Diese selten beobachtete Erkrankung wird vermutlich unterdiagnostiziert und ist durch kurze, dünne, langsam wachsende, nicht abbrechende Haare von Geburt bis ins frühe Kindesalter charakterisiert. In manchen Fällen wird berichtet, die Haare seien seit Geburt nicht geschnitten worden [45]. Der Pull-Test ist im Gegensatz zum Losen-Anagen-Haar-Syndrom negativ, und lichtmikroskopisch finden sich im Trichogramm Haarschäfte von dünnem Kaliber ohne wesentliche Strukturstörungen sowie eine erhöhte Telogenrate. Durch eine verkürzte Anagenphase ist das Längenwachstum nur zeitlich verkürzt möglich, sodass ein früher Übertritt in die Telogenphase stattfindet, was zu einer gering erhöhten Lichtung der betroffenen Areale führen kann [46].

Therapie. Der gestörte Haarzyklus kann sich während der Pubertät normalisieren. Therapeutisch kann aber auch ein Behandlungsversuch mit Minoxidil, worunter teilweise gute Erfolge berichtet wurden, unternommen werden [47].

Das Short-Anagen-Syndrom ist gekennzeichnet durch dünne, langsam wachsende, nicht abbrechende Haare. Der Pull-Test ist negativ.

\section{Diagnostik}

Eine detaillierte Anamnese mit exaktem Herausarbeiten des Beginns, der Anfangs- und VerlaufsDynamik des Haarausfalls sowie Ansprechbarkeit auf möglicherweise bereits durchgeführte Therapien ist Kernstück einer guten klinischen Diagnostik. Dies ist umso wichtiger, da Alopezien häufig schleichend beginnen und ein exakter Zeitpunkt des Beginns durch den Patienten häu- fig nicht leicht zu benennen ist. Von wesentlicher Bedeutung ist die Zeitdynamik v. a. zur Diagnosestellung einer symptomatischen diffusen Alopezie bei Assoziation mit Medikamenteneinnahme, Traumata, Neuauftreten von Mangelerscheinungen oder Systemerkrankungen. Die klinische Untersuchung des Kapillitiums sollte stehend am sitzenden Patienten unter quasi „Kartierung“ aller Kopfareale (temporal, parietal, okzipital jeweils beidseits, Scheitelregion, Vertex) unter Durchführung des Pull-Tests (Zupftest) und zusätzlich durch klinisch-dermatoskopische Begutachtung der Kopfhaut erfolgen. Schuppenpräparate bzw. bakterielle Abstriche sind bei klinischem Verdacht auf Tinea bzw. bakterielle Follikulitis durchzuführen. Zur Akuität der Alopezie bzw. Abgrenzung diffus beginnender androgenetischer Alopezie der Frau gegenüber symptomatischer diffuser Alopezie und zur Diagnostik von Losem-Anagen-Haar-Syndrom und Short-Anagen-Syndrom kann die Durchführung eines Trichogrammes sinnvoll sein. Zur Bestimmung der Haardichte (Haaranzahl $\mathrm{n} / \mathrm{cm}^{2}$ ) und des AnagenTelogen- und Vellus-Terminalhaaranteils steht zur Erstellung eines Ausgangsbefundes und zum Prä-post-Vergleich das TrichoScan, eine softwaregestützte digitale Auflichtmikroskopie von Haaren, als objektive Messmethode zur Verfügung $[48,49]$. Eine Kopfhautbiopsie sollte bei Verdacht auf vernarbende Alopezien aus technischen Gründen immer als ausreichend große Spindelbiopsie (ca. 1,5×0,5 cm Größe) durchgeführt werden, da einerseits nur durch eine Skalpellbiopsie die sichere Tiefe bis in die Subkutis (Sitz der Anagenhaarwurzel) erreicht werden kann als auch andererseits nur durch eine ausreichende Horizontalausdehnung der Biopsie das repräsentative Vollbild der Entzündung bzw. Vernarbung optimal untersucht werden kann. Auch wegen einer parallel durchzuführenden Immunfluoreszenz ist eine Spindelbiopsie zur Teilung des Gewebes sinnvoll. Bei der Labordiagnostik ist ein Kosten-Nutzen-orientiertes Basisprogramm zu empfehlen, welches Blutbild, Eisen, Ferritin, ANAs, CRP, TSH, Prolaktin und evtl. HbA1c umfasst. Bei klinischem Verdacht auf weitere Störungen sind dementsprechende Parameter zu bestimmen. Vor allem ein Hormonstatus ist nur zur Bestätigung bzw. zur Subspezifizierung hormoneller Störungen zu erheben, wenn klinisch-anamnestisch Veränderungen (z.B. Androgenisierungserscheinungen, Cushingoider Habitus) bereits fassbar sind, da die Wahrscheinlichkeit eines Zufallsbefundes bei Absenz von klinischen Symptomen extrem gering ist.

\section{Zusammenfassung \\ $\nabla$}

Alopezien können grundsätzlich in reversible und nicht reversible Formen eingeteilt werden. Die häufigste Form einer potenziell reversiblen

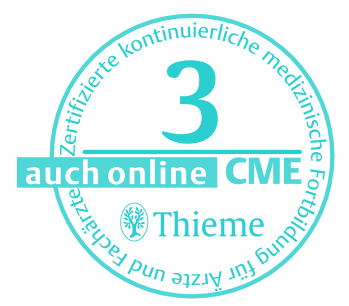


Alopezie ist die androgenetische Alopezie, die etwa jeden zweiten Mann und $10-20 \%$ der Frauen betrifft. Beim Mann liegt pathogenetisch eine erhöhte $5 \alpha$-Reduktase-Aktivität in genetisch prädeterminierten Haarfollikeln der Temporal- und Vertex-Region sowie eine erhöhte Expression des Androgen-Rezeptors zugrunde, während bei der Frau eine verminderte Aromatase-Aktivität im Vordergrund steht. Zur Behandlung stehen die systemische Anwendung von Finasterid beim Mann und die topische Anwendung von Minoxidil sowie $17 \alpha$ - und $17 \beta$-Estradiol beim Mann bzw. bei der Frau zur Verfügung.

Die zweithäufigste Form des Haarausfalls ist die Alopecia areata, die auf einer TH-1-gesteuerten autoimmunen Reaktion der Haarfollikel beruht und sich von einer einmalig auftretenden Episode mit einem singulären alopezischen Areal bis zur totalen Alopezie nicht nur der Kopfhaut, sondern auch der Augenbrauen, Wimpern, des Bartes, der Achsel- und Schambehaarung entwickeln kann. Die Behandlung erfolgt stadiengerecht mit topischen bzw. systemischen Kortikosteroiden und $\mathrm{ab}$ einer betroffenen Fläche von $>30 \%$ mit topischer Immuntherapie.

Die diffuse Alopezie ist ein klinischer Sammelbegriff von Alopezien, die ohne Bevorzugung von bestimmten Regionen das gesamte Kapillitium in mehr oder weniger starkem Ausmaß betreffen und - durch systemische Veränderungen (hormonell, stoffwechselbedingt, traumatisch, medikamentös) bedingt - symptomatisch werden. Die Identifikation der auslösenden Faktoren ist der erste Schritt zur Therapie, welche aus der Vermeidung einer Noxe bzw. Substitution von Mangelzuständen oder Therapie einer bestehenden Grunderkrankung besteht. Zu den irreversiblen Alopezien gehören der Lichen ruber follicularis, die postmenopausale frontal-fibrosierende Alopezie Kossard, der Lupus erythematodes capillitii, die Folliculitis decalvans, Pseuropelade Brocq und die zirkumskripte Sklerodermie „en coup de sabre“. In der Regel ist das Therapieziel dieser Alopezieformen, die entzündliche Reaktion und die fortschreitende Vernarbung zu reduzieren bzw. aufzuhalten. Dies erfolgt je nach Ausprägung mit topischen bzw. systemischen Kortikosteroiden, mit Retinoiden (Lichen ruber follicularis), mit Chloroquin (Lupus erythematodes capillitii) und mit Dapson bzw. bei der Folliculitis decalvans mit Antibiogramm-adaptierten systemischen Antibiotika. Im ausgebrannten Zustand (État pseudopeladique) sind befundgerechte kosmetische Maßnahmen zu wählen.

Seltene Erkrankungen sind das Lose-AnagenHaar-Syndrom, bei dem es durch eine Differenzierungsstörung der inneren Wurzelscheide zu einer Instabilität zwischen Wurzelscheide und Haarschaft mit leicht zu epilierenden Haaren kommt, während beim Short-Anagen-Syndrom die Wachstumsphasen (Anagen) zugunsten von längeren Ruhephasen (Telogen) verkürzt sind und kurze, langsam wachsende Haare resultieren. Beide Erkrankungen können nicht kausal behandelt werden, bessern sich aber in einigen Fällen mit zunehmendem Lebensalter bzw. können mit Minoxidil teilweise erfolgreich behandelt werden.

Die Diagnostik erfolgt bei der AGA, AA und AD anamnestisch und klinisch und bei Bedarf gestützt durch Labordiagnostik und in den Fällen von entzündlich-vernarbenden Alopezien durch ausreichend große Kopfhautbiopsien. Insgesamt ist die Patientenklientel der Alopezien durchaus schwierig und ärztlich anspruchsvoll, da Therapien aufgrund des langsamen Wachstums des Haares auch nur langsam Erfolge zeigen können und auch nur dann, wenn diese kontinuierlich durchgeführt werden und somit die Etablierung eines hochqualitativen Arzt-Patienten-Behandlungsbündnisses eine conditio sine qua non darstellt, um eine optimale langfristige PatientenCompliance und somit die bestmöglichen Therapieerfolge zu erreichen.

\section{Literatur}

1 Schmidt S, Fischer TW, Chren MM, Strauss BM, Elsner P. Strategies of coping and quality of life in women with alopecia. Br J Dermatol 2001; 144: 1038 - 1043

2 Fischer TW, Schmidt S, Strauss B, Elsner P. Hairdex - ein Instrument zur Untersuchung der krankheitsbezogenen Lebensqualität bei Patienten mit Haarerkrankungen. Hautarzt 2001; 52: 219-227

3 Hoffmann R, Happle R. Current understanding of androgenetic alopecia. Part I: etiopathogenesis. Eur J Dermatol 2000; 10: 319-327

4 Kaufman KD. Androgen metabolism as it affects hair growth in androgenetic alopecia. Dermatol Clin 1996; 14: $697-711$

5 Paus R, Cotsarelis $G$. The biology of hair follicles. N Engl ] Med 1999; 341: 491-497

6 Rebora A. Pathogenesis of androgenetic alopecia. J Am Acad Dermatol 2004; 50: 777-779

7 Trueb RM. Molecular mechanisms of androgenetic alopecia. Exp Gerontol 2002; 37: 981- 990

8 Foitzik K, Paus R. Regulation of human hair growth and therapeutic options. Journal of the German Society of Dermatology 2005; 3: 901-915

9 Fischer TW, Paus R. Androgenetic Alopecia. In: Encyclopedia of Molecular Mechanisms of Disease. Berlin: Springer, 2006

10 Hamilton JB. Patterned loss of hair in man: types and incidence. Ann N Y Acad Sci 1951; 53: 708-728

11 Norwood OT. Male-pattern baldness. Classification and incidence. South Med J 1975; 68: 1359-1370

12 Ludwig E. Classification of the types of androgenetic alopecia (common baldness) occurring in the female sex. Br J Dermatol 1977; 97: 247-254

13 Wolff $H$, Kunte C. Diagnostik und Therapie von Haarerkrankungen, Vol. 1. Bremen: Uni-Med, 1999

14 Bergner T, Braun-Falco O. Die androgenetische Alopezie der Frau. Hautarzt 1991; 42: $201-210$

15 Schweikert HU, Wilson JD. Regulation of human hair growth by steroid hormones. I. Testerone metabolism in isolated hairs. J Clin Endocrinol Metab 1974; 38: $811-819$

16 Kaufman KD, Olsen EA, Whiting D, Savin R, De Villez $R$, Bergfeld W, Price VH, Van Neste D, Roberts JL, Hordinsky M, Shapiro J, Binkowitz B, Gormley GJ. Finasteride in the treatment of men with androgenetic alopecia. Finasteri- 
de male pattern hair loss study group. J Am Acad Dermatol 1998; 39: 578-589

17 Jacobs JP, Szpunar CA, Warner ML. Use of topical minoxidil therapy for androgenetic alopecia in women. Int J Dermatol 1993; 32: 758-762

18 Olsen EA, Dunlap FE, Funicella T, Koperski JA, Swinehart JM, Tschen EH, Trancik RJ. A randomized clinical trial of $5 \%$ topical minoxidil versus $2 \%$ topical minoxidil and placebo in the treatment of androgenetic alopecia in men. J Am Acad Dermatol 2002; 47: 377-385

19 Whiting DA, Jacobson C. Treatment of female androgenetic alopecia with minoxidil 2\%. Int J Dermatol 1992; 31: 800-804

20 Lucky AW, Piacquadio DI, Ditre CM, Dunlap F, Kantor I, Pandya AG, Savin RC, Tharp MD. A randomized, placebo-controlled trial of $5 \%$ and $2 \%$ topical minoxidil solutions in the treatment of female pattern hair loss. J Am Acad Dermatol 2004; 50: 541 - 553

21 Olsen EA, Whiting D, Bergfeld W, Miller J, Hordinsky M, Wanser $R$, Zhang P, Kohut B. A multicenter, randomized, placebo-controlled, double-blind clinical trial of a novel formulation of $5 \%$ minoxidil topical foam versus placebo in the treatment of androgenetic alopecia in men. J Am Acad Dermatol 2007; 57: 767 - 774

22 Freyschmidt-Paul P. Alopecia areata. CME Dermatologie 2007; 3: 4- 14

23 Tosti A, Iorizzo M, Botta GL, Milani M. Efficacy and safety of a new clobetasol propionate $0.05 \%$ foam in alopecia areata: a randomized, double-blind placebo-controlled trial. J Eur Acad Dermatol Venereol 2006; 20: $1243-$ 1247

24 Fischer TW. Haarausfall bei internmedizinischen Erkrankungen. Therapeutische Umschau 2002; 59: 238- 242

25 Hendrix S, Handjiski B, Peters EM, Paus R. A guide to assessing damage response pathways of the hair follicle: lessons from cyclophosphamide-induced alopecia in mice. J Invest Dermatol 2005; 125: 42-51

26 Schell H. Erkrankungen der Haare, Vol. 1. Stuttgart, Berlin, Köln: W. Kohlhammer, 1997

27 Fischer $T$, Köhler LD, Vogt $\mathrm{H}-J$. Haarveränderungen durch Krankheit oder Medikamente. Hautnah Dermatologie 2000; 5: 7-11

28 Graham-Little E. Folliculitis decalvans et atrophicans. Proc Roy Soc Med 1915; 8: 139-140

29 Kossard S. Postmenopausal frontal fibrosing alopecia. Scarring alopecia in a pattern distribution. Arch Dermatol 1994; 130: $770-774$

30 Moreno-Ramirez D, Camacho Martinez F. Frontal fibrosing alopecia: a survey in 16 patients. J Eur Acad Dermatol Venereol 2005; 19: 700 - 705

31 Kossard S, Lee MS, Wilkinson B. Postmenopausal frontal fibrosing alopecia: a frontal variant of lichen planopilaris. J Am Acad Dermatol 1997; 36: 59-66
32 Tosti A, Piraccini BM, Iorizzo M, Misciali C. Frontal fibrosing alopecia in postmenopausal women. J Am Acad Dermatol 2005; 52: 55-60

33 Zinkernagel MS, Trueb RM. Fibrosing alopecia in a pattern distribution: patterned lichen planopilaris or androgenetic alopecia with a lichenoid tissue reaction pattern? Arch Dermatol 2000; 136: 205-211

34 Wilson CL, Burge SM, Dean D, Dawber RP. Scarring alopecia in discoid lupus erythematosus. $\mathrm{Br}$ J Dermatol 1992; 126: $307-314$

35 Hermes B, Paus $R$. „Vernarbende“ Alopezien - Anmerkungen zur Klassifikation, Differenzialdiagnose und Pathobiologie. Hautarzt 1998; 49: 462-472

36 Braun-Falco O, Bergner T, Heilgemeir GP. The Broca pseudopelade - a disease picture or disease entity. Hautarzt 1989; 40: 77-83

37 Paquet P, Pierard GE. Dapsone treatment of folliculitis decalvans. Ann Dermatol Venereol 2004; 131: 195-197

38 Abeck D, Korting HC, Braun-Falco O. Folliculitis decalvans. Long-lasting response to combined therapy with fusidic acid and zinc. Acta Derm Venereol 1992; 72: 143-145

39 Brooke RC, Griffiths CE. Folliculitis decalvans. Clin Exp Dermatol 2001; 26: 120-122

40 Powell J, Dawber RP. Successful treatment regime for folliculitis decalvans despite uncertainty of all aetiological factors. Br J Dermatol 2001; 144: 428-429

41 Brocq L. Des folliculites et perifolliculites decalvantes. Bull Mem Soc Med Hop Paris 1988; 5: 399-408

42 Price VH, Gummer CL. Loose anagen syndrome. J Am Acad Dermatol 1989; 20: 249-256

$43 \mathrm{Hamm} \mathrm{H}$, Traupe H. Loose anagen hair of childhood: The phenomenon of easily pluckable hair. I Am Acad Dermatol 1989; 20: $242-248$

44 Tosti A, Peluso AM, Misciali C, Venturo N, Patrizi A, Fanti PA. Loose anagen hair. Arch Dermatol 1997; 133: 10891093

45 Antaya RJ, Sideridou E, Olsen EA. Short anagen syndrome. J Am Acad Dermatol 2005; 53: 130 - 134

46 Barraud-Klenovsek MM, Trueb RM. Congenital hypotrichosis due to short anagen. $\mathrm{Br}$ ] Dermatol 2000; 143: 612-617

47 Van Egmond S, Hoedemaker C, Sinclair R. Short anagen hair naevus: improvement after treatment with $5 \%$ topical minoxidil. Int J Dermatol 2007; 46: 757 - 759

48 Hoffmann R. TrichoScan: combining epiluminescence microscopy with digital image analysis for the measurement of hair growth in vivo. Eur J Dermatol 2001; 11: $362-368$

49 Hoffmann $R$. TrichoScan, a GCP-validated tool to measure hair growth. J Eur Acad Dermatol Venereol 2008; 22: 132 - 134; author reply $134-135$

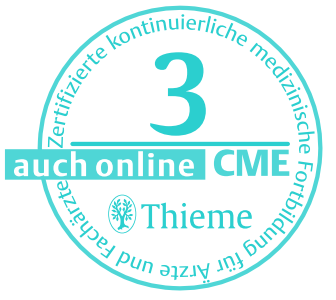




\section{CME-Fragen Alopezien - Diagnostisches und therapeutisches Management}

Welche Ursache bzw. welche Erkrankung kann am wahrscheinlichsten vorliegen, wenn bei einer Frau eine völlige Kahlheit der Kopfhaut besteht?

1 Zustand nach Chemotherapie

2 androgenetische Alopezie „female pattern“

3 frontal-fibrosierende postmenopausale Alopezie Kossard

4 Alopecia areata

5 diffuses Effluvium nach schwerem grippalem Infekt mit sekundär bakterieller Pneumonie

A Nur die Aussage 1 ist richtig.

B Nur die Aussagen 2 und 4 sind richtig.

C Nur die Aussagen 1 und 4 sind richtig.

D Nur die Aussagen 1, 4 und 5 sind richtig.

E Alle Aussagen sind richtig.

Was bezeichnet man als „Clown-Alopezie“?

die Maximalform der androgenetischen Alopezie „female pattern" wenn nur noch eine frontale Haarlinie und ein okzipitaler Haarkranz besteht

B eine besondere klinische Ausprägung der Alopecia areata

C eine andere Bezeichnung für die Alopecia areolaris specifica bei Lues

D ein Begleitsymptom bei Harlekin-Fetus

E die fortgeschrittene Form der postmenopausalen frontalfibrosierenden Alopezie Kossard

Für die Alopecia areata trifft folgende Aussage zu:

A Es findet sich ein dichtes leukozytäres Zellinfiltrat um den Haarbulbus.

B Die Alopecia areata ist eine potenziell reversible Alopezie.

C Der Ophiasis-Typ ist durch eine gute Prognose gekennzeichnet.

D Bei Manifestation im frühen Kindesalter sind häufig schwere Verläufe zu beobachten.

E Kortikosteroide führen häufig zu einer Remission.

Welche Antwort zum dystrophen Frühtyp und verzögerten Spättyp der diffusen Alopezie trifft nicht zu? Chemotherapie induziert typischerweise ein Effluvium vom Frühtyp.

B Sowohl der Frühtyp als auch der Spättyp ist reversibel.

C Beim akuten Frühtyp treten alle Anagen-Follikel sofort in das Telogen-Stadium über.

D Beim Spättyp werden durch die Noxe alle Haarfollikel gleichzeitig in das Katagen- und Telogen-Stadium getrieben.

E Das Effluvium vom Spättyp manifestiert sich innerhalb von Wochen bis Monaten.
Welche Merkmale finden sich beim Lichen ruber follicularis in der direkten Immunfluoreszenz? 1 lamellar bodies

2 cytoid bodies

3 netzartiges Fluoreszenzmuster

4 Papillenabszesse

5 vaskuläre Fibrinablagerungen

A Nur Aussage 1 ist richtig.

B Nur die Aussagen 2 und 4 sind richtig.

C Nur die Aussagen 2 und 5 sind richtig.

D Nur die Aussagen 2, 4 und 5 sind richtig.

E Alle Aussagen sind richtig.

6 Die postmenopausale frontal-fibrosierende Alopezie Kossard ist in wieviel Prozent der Fälle mit androgenetischer Alopezie vergesellschaftet?

A in ca. $12,5 \%$ der Fälle

B in ca. $24 \%$ der Fälle

C in ca. $35 \%$ der Fälle

D in ca. $40 \%$ der Fälle

E in ca. $46 \%$ der Fälle

In wieviel Prozent der Fälle finden sich beim Lupus erythematodes capillitii antinukleäre Antikörper? in $90 \%$ der Fälle

in $70 \%$ der Fälle

in $50 \%$ der Fälle

in $30 \%$ der Fälle

in $20 \%$ der Fälle

Die Pseudopelade Brocq ist wie folgt charakterisiert:

1 Das klinische Bild ist durch fleckartige, fibrotische Areale mit Destruktion der Haarfollikel gekennzeichnet.

2 Histologisch findet sich ein ausgeprägtes spezifisches CD4+-/CD8+-Zellinfiltrat.

3 Die Histologie zeigt weniger Entzündungsinfiltrat als der Lupus erythematodes capillitii und der Lichen ruber follicularis.

4 Klinisch kann die Pseudopelade Broqc vom Lupus erythematodes capillitii durch ein stärker ausgeprägtes, scharfes, randbetontes entzündliches Erythem unterschieden werden.

5 Die Pseudopelade Broqc und der État pseudopeladique sind synonym zu gebrauchen.

Nur die Aussage 1 ist richtig.

Nur die Aussagen 1 und 5 sind richtig.

Nur die Aussagen 1 und 3 sind richtig.

Nur die Aussagen 2 und 4 sind richtig.

Nur die Aussagen 2, 4 und 5 sind richtig. 
Beim Losen-Anagen-Haar-Syndrom finden sich folgende Merkmale:

1 Funktionell ist ein loser Sitz der dermalen Papille des Anagenhaares ursächlich.

2 Die gestörte Keratinisierung der inneren Wurzelscheide spielt eine ursächliche Rolle.

3 Der Pull-Test ist negativ.

4 Das Lose-Anagen-Haar-Syndrom tritt im Zuge der Seneszenz der Haarfollikel mit zunehmendem Lebensalter auf.

5 Das Lose-Anagen-Haar-Syndrom tritt bei Kindern auf. Nur die Aussage 1 ist richtig.

Nur die Aussagen 1 und 2 sind richtig. Nur die Aussagen 1, 2, 3 und 4 sind richtig. Nur die Aussagen 1, 2 und 5 sind richtig. Nur die Aussagen 2 und 5 sind richtig.
Welche Aussagen zum Short-Anagen-Syndrom sind richtig?

1 Es findet sich ein negativer Pull-Test.

2 Die Haare brechen aufgrund von Fragilität leicht ab.

3 Lichtmikroskopisch finden sich keine Strukturschäden.

4 Im Trichogramm finden sich vermehrt Telogenhaare. 5 Therapeutisch kann Minoxidil hilfreich sein.

A Nur die Aussage 1 ist richtig.

B Nur die Aussagen 1, 2 und 3 sind richtig.

C Nur die Aussagen 1, 3 und 4 sind richtig.

D Nur die Aussagen 1, 3, 4 und 5 sind richtig.

E Alle Aussagen sind richtig. 
A Angaben zur Person

Name, Vorname, Titel:
Straße, Hausnr.:

Anschrift: $\quad$ privat
EFN-Nummer:
Ich bin Mitglied der Ärztekammer (bitte Namen der Kammer eintragen):
Jahr meiner Approbation:
Ich befinde mich in der Weiterbildung zum:
Ich habe eine abgeschlossene Weiterbildung in (bitte Fach eintragen):
Ich bin tätig als: Assistenzarzt Oberarzt Chefarzt $\quad$ niedergelassener Arzt Sonstiges:

\section{B Lernerfolgskontrolle}

Bitte nur eine Antwort pro Frage ankreuzen

\section{1}

2

\section{3}

4

5 A

1 A $B$ B $C$ D

2 A

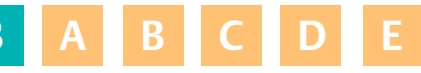

4 A

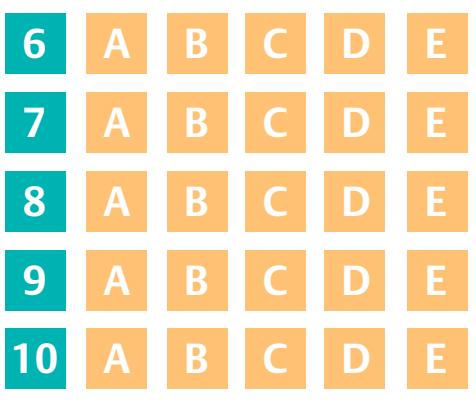

6 A $B$ B

7 A

8 A $\quad$ B $\quad$ C

\begin{tabular}{l|l|l|l|l|l}
9 & $\mathrm{~A}$ & $\mathrm{~B}$ & $\mathrm{C}$ & $\mathrm{D}$ & $\mathrm{E}$
\end{tabular}

10 A $B$ C

Erklärung

Ich versichere, dass ich die Beantwortung der Fragen selbst und ohne Hilfe durchgeführt habe

Ort | Datum:

Feld für CME-Wertmarke

Bitte in dieses Feld die CME-Wertmarke kleben oder Ihre Abonnement-Nummer eintragen:

(siehe Adressaufkleber)

Zertifizierungsfeld (wird durch den Verlag ausgefüllt)
Unterschrift:

E Zertifizierungsfeld

\section{Ihr Ergebnis}

Sie haben von 10 Fragen richtig beantwortet.

Sie haben

bestanden und 3 CME-Punkte erworben.

nicht bestanden

Stuttgart, den

Datum

Stempel/Unterschrift

Bitte unbedingt Rückseite ausfüllen! 


\section{F Fragen zur Zertifizierung}

Eine Antwort pro Frage. Bitte unbedingt ausfüllen bzw. ankreuzen, da die Evaluation sonst unvollständig ist!

\section{Didaktisch-methodische Evaluation}

1 Das Fortbildungsthema kommt in meiner ärztlichen Tätigkeit

häufig vor

regelmäßig vor

selten vor

gar nicht vor

Zum Fortbildungsthema habe ich

eine feste Gesamtstrategie

noch offene Einzelprobleme:

keine Strategie

3 Hinsichtlich des Fortbildungsthemas

fühle ich mich nach dem Studium des Beitrags in meiner Strategie bestätigt

habe ich meine Strategie verändert:

habe ich erstmals eine einheitliche Strategie erarbeitet

habe ich keine einheitliche Strategie erarbeiten können

4 Wurden aus der Sicht Ihrer täglichen Praxis heraus wichtige Aspekte des Themas

\begin{tabular}{l|l|l|l|}
\hline nicht erwähnt? & ja, welche & nein \\
\hline zu knapp behandelt? & ja, welche & nein \\
\hline überbewertet? & ja, welche & nein \\
\hline
\end{tabular}

5 Verständlichkeit des Beitrags

Der Beitrag ist nur für Spezialisten verständlich

Der Beitrag ist auch für Nicht-Spezialisten verständlich

6 Beantwortung der Fragen

Die Fragen lassen sich aus dem Studium des Beitrages allein beantworten

Die Fragen lassen sich nur unter Zuhilfenahme zusätzlicher Literatur beantworten

7 Die Aussagen des Beitrages benötigen eine ausführlichere Darstellung

zusätzlicher Daten

von Befunden bildgebender Verfahren

die Darstellung ist ausreichend

8 Wie viel Zeit haben Sie für das Lesen des Beitrages und die Bearbeitung des Quiz benötigt? 